\title{
Rat Proliferative Change by Location
}

National Cancer Institute

\section{Source}

National Cancer Institute. Rat Proliferative Change by Location. NCI Thesaurus. Code C61111.

Disorders of the rat that are characterized by cell proliferation, organized by body location. 